\title{
Approach to the Diagnostic Workup and Management of Small Bowel Lesions at a Tertiary Care Center
}

Stein $\mathrm{D}^{1}$, Green $\mathrm{J}^{1}$, Schlieve $\mathrm{CR}^{1}$, Friedrich AK², Baratta K², Ma DH${ }^{1}$, Min $\mathrm{M}^{1}$, Patel K${ }^{1}$, Cave DR ${ }^{1}$, Litwin DE${ }^{1}$, Cahan MA

1 University of Massachusetts Medical School, 55 Lake Avenue North, Worcester MA 01655

2 Saint Mary's Hospital, 56 Franklin Street, Waterbury, Connecticut, 06706

\section{Background and Purpose}

Primary small bowel lesions (SBLs) are difficult to diagnose with modern endoscopic and radiographic techniques.

- SBLs are rare, yet vary widely in pathology and gastrointestinal tract location.

- It is important to review prior SBL cases to understand what factors were most beneficial in directing diagnostic approaches and subsequent treatments for these patients.

- Here, we evaluate diagnostic modalities and management practices of patients with SBLs at an advanced endoscopic referral center.

\section{Methods}

- We analyzed patients undergoing surgical management for SBL from 2005-2015 at a single tertiary care center.

- Patients were stratified into two major presenting symptoms and signs: Gastrointestinal bleed/anemia (GIBA) or obstruction/pain (OP).

\section{Results Summary}

112 patients underwent surgery after presenting with GIBA $(n=67)$ or OP $(n=45)$.

- Mean age was 61.8 years and $45 \%$ were women

- Patients with GIBA had a higher body mass index, were more likely to have chronic or acute-on-chronic symptoms $(100 \%$ vs. $67 \%)$, and more often referred from outside hospitals ( $82 \%$ vs. $44 \%)(p<0.01)$.

- Most common preoperative imaging modalities were video capsule endoscopy (VCE) $(96 \%)$ for GIBA and computer tomography CT $(78 \%)$ for OP.

- VCE and CT findings were frequently concordant with operative findings in GIBA $(67 \%)$ and OP (54\%) patients, respectively.

- Intraoperative visual inspection or palpation of the bowel identified lesions in $71 \%$ of patients.

- Intraoperative Enteroscopy confirmed or identified lesions in $69 \%$ of GIBA patients $(n=26)$

- $90 \%$ of GIBA patients had a small bowel resection (93\% laparoscopic)

- $58 \%$ of OP patients had a small bowel resection ( $81 \%$ laparoscopic)

- Surgical exploration failed to identify lesions in $10 \%$ of GIBA patients and $24 \%$ of OP patients.

- $20 \%$ of GIBA patients (vs. $13 \%$ of OP patients) who underwent resections had recurrent symptoms

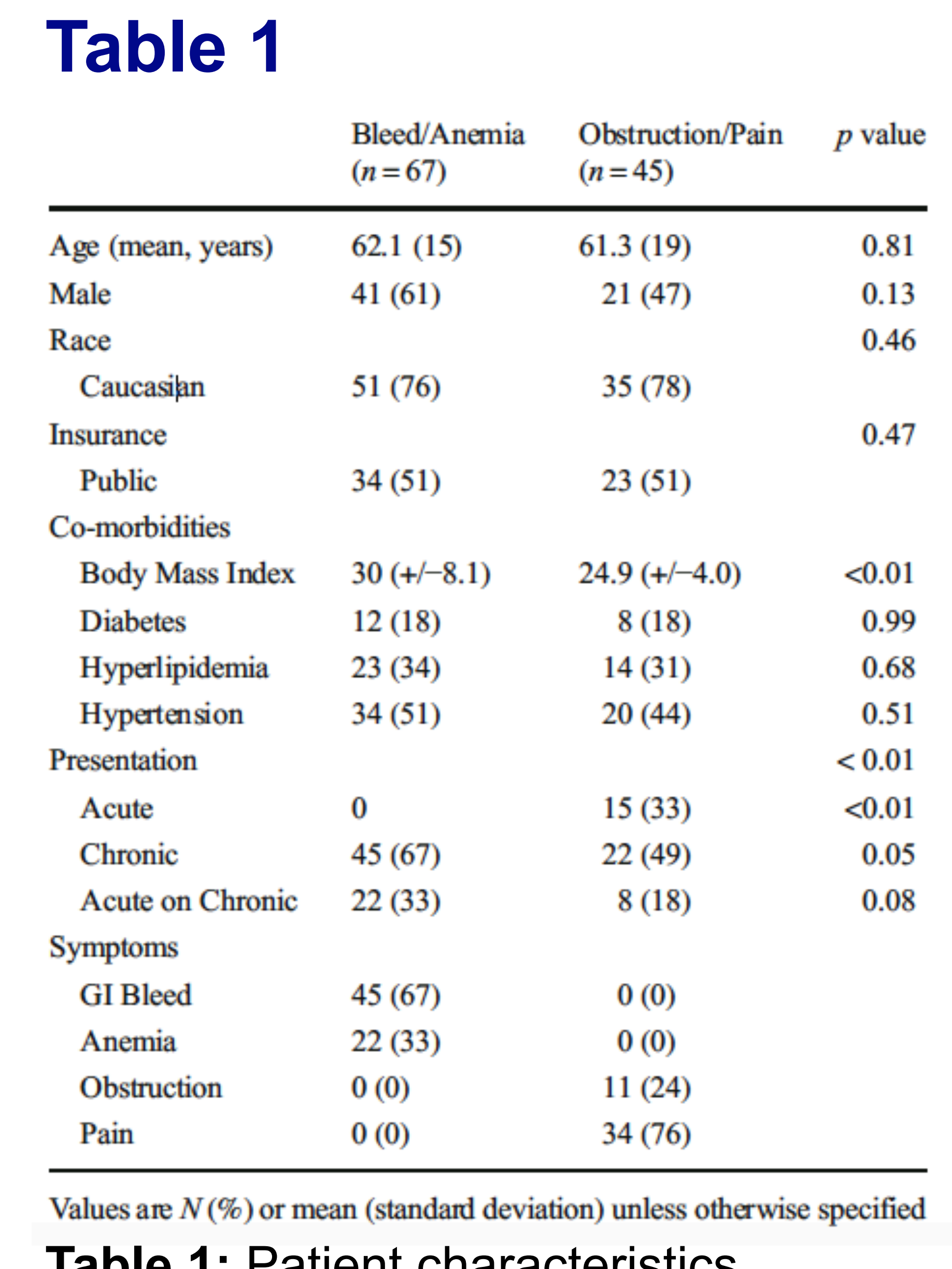

Table 1: Patient characteristics according to clinical presentation

Figure 1

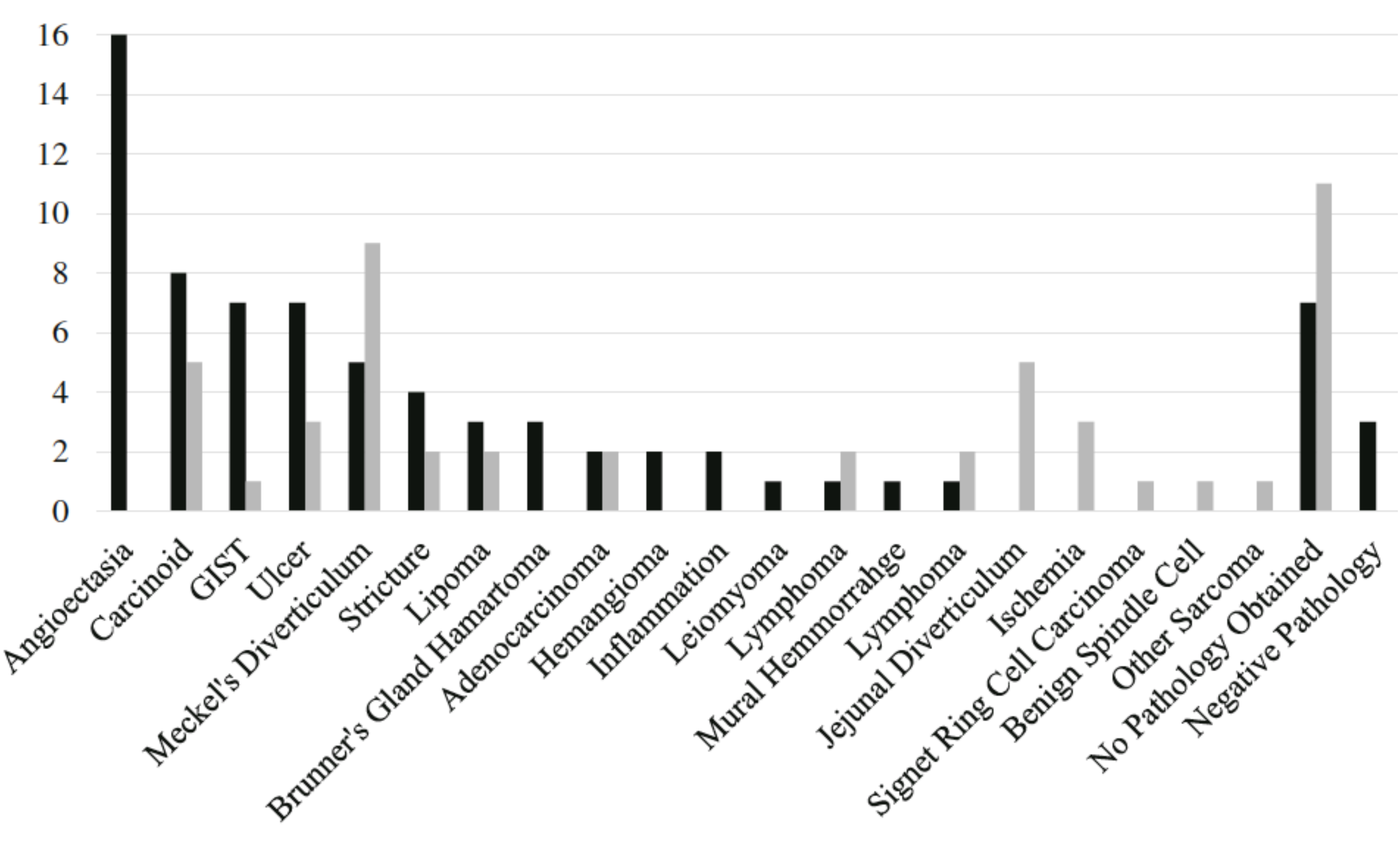

$\operatorname{GIBA}(\mathrm{n}=67) \quad \square \mathrm{OP}(\mathrm{n}=45)$

Figure 1: A graphical representation of pathology based on clinical presentation. Gastrointestinal bleed and anemia (GIBA) area represented in black. Obstruction and pain are represented in gray. Values are not mutually exclusive.

\section{Conclusions}

- Management and identification of SBL is governed by presenting symptomatology

- Optimal management includes VCE and IE for GIBA and CT scans for OP patients.

- Comprehensive evaluation may require referral to specialized centers

\section{Acknowledgements}

I would like to thank all authors and staff in the Surgery and Gastroenterology departments at UMass Medical School, and the Senior Scholars Program for the opportunity to showcase this study.
Figure 2: Approach to the management of suspected small bowe symptoms of either treatment should be determined by pIIBA) or obstruction/pain (OP). Deep enteroscopy (DE) includes spiral enteroscopy (SBE), and double-balloon ( Computed tomography (CT), computed tomography enterography (CTE), intraoperative enteroscopy (IE)

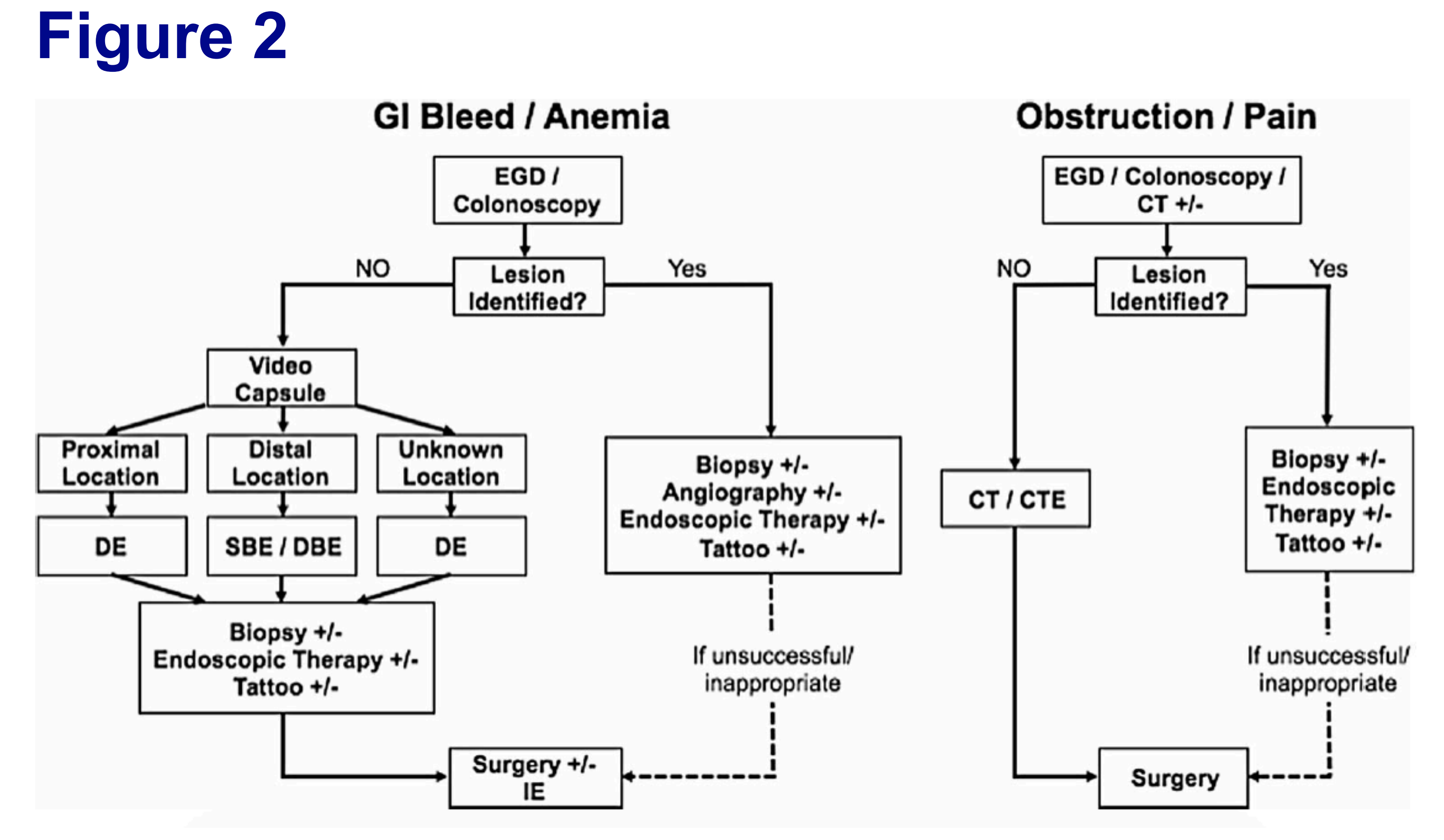

